\title{
DESIGN OF LONGITUDINAL FEEDBACK SYSTEM KICKER FOR THE PLS STORAGE RING*
}

\author{
Yujong Kim, M. Kwon, I. S. Park, $\underline{\mathrm{I} . \mathrm{S} . \mathrm{Ko}}^{\dagger}$, PAL, POSTECH, Pohang 790-784, Korea
}

\section{Abstract}

The higher order modes (HOMs) of RF cavities at the Pohang Light Source (PLS) storage ring cause longitudinal coupled bunch mode instabilities (CBMIs). To cure these instabilities, a longitudinal feedback system (LFS) is introduced. As a key component of the LFS, there is a single-ridged waveguide-overloaded cavity as a longitudinal bunch-by-bunch LFS kicker for the PLS storage ring. To damp any coupled bunch modes, the bandwidth of this kicker should be wider than $250 \mathrm{MHz}$. Also, the higher shunt impedance of the kicker is selected to use lower power amplifier. One aluminum kicker is fabricated and the bandwidth, HOMs, and shunt impedance of the kicker are measured with a network analyzer. This kicker has several different features from LFS kicker for DA $\Phi N E$. First of all, there are 4 input/output ports to obtain a wider bandwidth. Secondly, there is a nose cone to obtain higher shunt impedance. Finally, the symmetric frequency response of the shunt impedance around central frequency is provided. According to the simulation result done by HFSS code, high shunt impedance of $620 \Omega$ (transit time factor considered value) and the wide bandwidth of $255 \mathrm{MHz}$ are obtained. These are compared with the measured result.

\section{INTRODUCTION}

The PLS is the 3rd generation synchrotron light source. Originally, the PLS storage ring is designed to store the beam current up to $400 \mathrm{~mA}$ at $2 \mathrm{GeV}$ and $250 \mathrm{~mA}$ at 2.5 $\mathrm{GeV}$. By adding one RF cavity in 1996, there are four RF cavities with $60 \mathrm{~kW} \mathrm{CW}$ klystron amplifier operating at the PLS storage ring to store the desired beam current [1]. But owing to HOMs of RF cavities that make the CBMIs such as dipole, quadrupole, sextupole modes, the current of PLS storage ring is possible up to $200 \mathrm{~mA}$ at $2.0 \mathrm{GeV}$ without CBMI. By analyzing the reverse signal of RF cavities and the sidebands of the BPM spectrum, it is found that the most dangerous HOMs of RF cavities are longitudinal $\mathrm{TM}_{011}(758 \mathrm{MHz})$ and $\mathrm{TM}_{013}(1707 \mathrm{MHz})$ modes. To avoid these HOMs, the precession temperature control system for the RF cavities has been installed during 1997 Summer maintenance period. This system can regulate the cooling water to better than $0.2^{\circ} \mathrm{C}$. With this system, the stored beam current without CBMIs has been increased from 120 $\mathrm{mA}$ to $200 \mathrm{~mA}$. It means that the cooling system is not enough to avoid such dangerous HOMs fully. So, an active feedback system for CBMIs is necessary for the PLS. The typical LFS consists of a signal pickup, digital signal pro-

\footnotetext{
${ }^{*}$ Work supported by POSCO and MOST, Korea.

† Email : isko@ postech.ac.kr
}

cessing units, and a kicker. There are various types of LFS developed at several accelerator laboratories such as SLAC, LBL, Frascati, KEK, etc. In the PLS case, it is decided that the digital signal processing unit is purchased from SLAC and the kicker is fabricated by the PLS and domestic manufacturer. By considering these design, the PLS has decided to design the kicker following DA $\Phi$ NE design [2] that is a single-ridged waveguide-overloaded cavity with HFSS and SUPERFISH codes.

\section{DESIGN OF LFS KICKER}

There are many things to be considered in order to design the LFS kicker for the PLS storage ring such as kicker length, working central frequency, bandwidth, shunt impedance, kicker filling time, contents of HOMs, kicker input power or kicker amplifier power. The LFS kicker will be installed at the straight section for RF cavities where there is a space of $42 \mathrm{~cm}$ reserved for future fifth cavity. Thus, the kicker length is determined to be $42 \mathrm{~cm}$. According to CBMI theory, coupled bunch modes are characterized by a definite phase relation between the oscillations from one bunch to the next. Since all CBMIs are located within the frequency range of $p \cdot f_{R F} \sim(p+1 / 2) \cdot f_{R F}$ where $p$ is any integer, the minimum bandwidth of kicker to cure all CBMIs is $f_{R F} / 2$, and the central frequency of the kicker $f_{c}$ is the average of the frequency range, $(p+1 / 4) \cdot f_{R F}$. Next is the selection of central frequency which is determined by choosing an integer $p$. By considering commercially available amplifiers and the operating frequency of PLS RF system of $500 \mathrm{MHz}$, the possible choice of $p$ for the PLS LFS is either 2 or 3 , which corresponds the central frequency of $1125 \mathrm{MHz}$ or 1625 $\mathrm{MHz}$, respectively, and $p=2$ is selected. The selected RF amplifier is a solid state type model AS0820-250R from MILMEGA. Its operation frequency range is $800 \mathrm{MHz} \sim$ $2000 \mathrm{MHz}$ and maximum output is $250 \mathrm{~W}$. Even though the PLS LFS is designed to operate at $p=2$ mode with bandwidth of $250 \mathrm{MHz}$ and the central frequency of $1125 \mathrm{MHz}$, this amplifier will also be used at $p=3$ mode in the future for more efficient kicking with lower RF power. Because the maximum power of amplifier is limited, the amplitude of the correction kick will be saturated easily in damping the phase oscillation with very high amplitude (i.e., very fast instability growth rate). One way to lower the required power of the amplifier in damping the CBMIs is to design the kicker with high shunt impedance. Since the RF frequency of the PLS storage ring is $500 \mathrm{MHz}$ and the harmonic number is 468 , the bunch spacing in fully filled case is $2 \mathrm{nsec}$. To perform the bunch-by-bunch kicking properly, the filling time of LFS kicker must be shorter than 2 
Table 1: $f_{c}$ vs. other parameters

\begin{tabular}{lcc}
\hline Integer $p$ & 2 & 3 \\
\hline \hline Central frequency $f_{c}[\mathrm{MHz}]$ & 1125 & 1625 \\
Loaded Q factor $Q_{L}$ & 4.5 & 6.5 \\
Max. shunt impedance $R_{s}[\Omega]$ & 450 & 650 \\
Filling time $\tau$ [nsec] & 1.27 & 1.27 \\
\hline
\end{tabular}

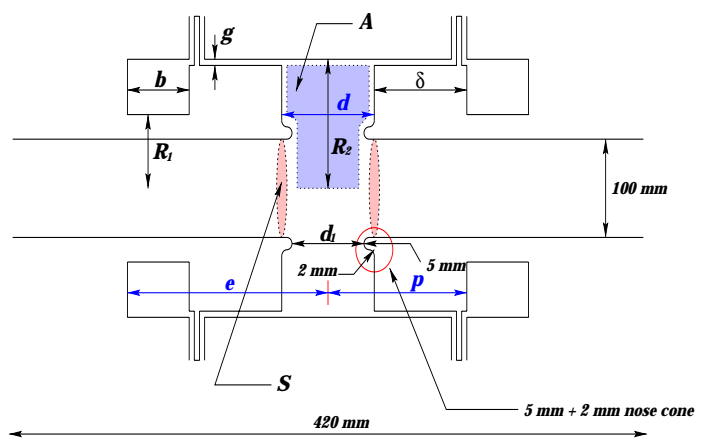

(a) Side view

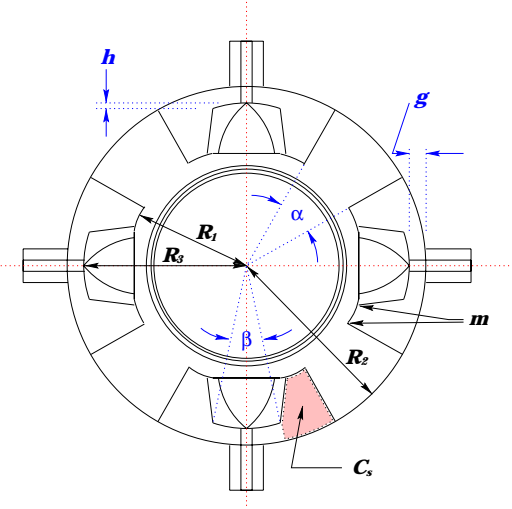

(b) Axial view

Figure 1: Side view and axial view of the PLS LFS kicker.

nsec. The relation between the central frequency and other parameters are summarized in Table 1.

\section{TUNING OF KICKER DIMENSION}

The DA $\Phi$ NE type kicker can be simulated with Ansoft 3D high frequency structure simulator (HFSS). Unlike the original DA $\Phi$ NE kicker, the PLS kicker is designed with a nose cone attached and 4 input/output ports. The profile of the PLS LFS kicker is shown in Fig. 1.

\subsection{Tuning of Basic Pill-Box Cavity}

It is well known that the electrical equivalence of a RF cavity is a series RLC circuit. Its resonance frequency $f_{r}$ is given by $f_{r}=1 / 2 \pi \sqrt{L C}$. The central frequency $f_{c}$ of RF cavity is equivalent to the resonance frequency $f_{r}$ of the RLC circuit. By adjusting dimensions of the kicker geometry that are equivalent counterparts of inductance $L$ and capacitance $C$, one can tune the central frequency of the kicker such as $f_{c} \sim 1 / R_{2}$ where $R_{2}$ is the outer radius of pill-box cavity. From the fact that the shunt impedance $R_{s}$ is proportional to $R / Q$, one must adjust the gap size (plate separation) $d$ and the outer radius of the kicker to obtain maximum $R / Q$. Since the central frequency $f_{c}$ is independent on the gap size $d, f_{c}$ is not changed by adjusting the gap size with constant $R_{2}$. But the shunt impedance is inversely proportional to bandwidth, too high shunt impedance will lead to too narrow bandwidth. Therefore, one must reduce the $R_{s}$ properly to have sufficient bandwidth. The cross section area of a waveguide covers about $11 \%$ of available surface of the pill-box side. If one attach the waveguide more, one can increase wall loss area, which means the increase (decrease) of total wall loss power (quality factor). To obtain a sufficient bandwidth (> $250 \mathrm{MHz}$ ), four waveguides are attached per pill-box side, so total waveguides are 8 ( 4 input/output ports). When the gap voltage or the shunt impedance of the kicker is considered, the transit time factor must be considered to correct the particle acceleration due to the time variation of the field while the particles traverse the kicker. In the case of constant frequency, the transit time factor $T$ increases as the gap size $d$ decreases and higher shunt impedance is possible by decreasing the gap size. When $d$ is fixed, the transit time factor will decrease as the frequency $f$. Therefore, at high frequency region, the shunt impedance will be lower than that of low frequency region around the central frequency. This problem of anti-symmetry is cured by changing the structure of ports from flat to round bases with $h=1 \mathrm{~mm}$, as shown in Fig. 1. After changing the geometry of port bases, anti-symmetry $S_{21}$ parameters around the central frequency are obtained. The $S_{21}$ values of $f>f_{c}$ region are higher than those of $f<f_{c}$ region. Because the transit time factor of $f>f_{c}$ region decreases as the frequency increases, one can obtain the frequency response symmetry in the shunt impedance. To reduce the power reflection at the transition between coaxial ports and waveguides, one must match the impedance at the transition. While there is a certain $\mathrm{TE}_{10}$ mode cutoff frequency at general rectangular waveguide, there is no cutoff frequency at the coaxial input port. Therefore, to match the impedance at the transition, one must lower the $\mathrm{TE}_{10}$ mode cutoff frequency. To do this, a single ridge is attached at the bottom of general waveguide because of the capacitive effect between the ridge and the outer cavity. The cutoff frequency of a single-ridged waveguide can be lowered further by decreasing the gap $g$ as shown in Fig. 1 .

\subsection{Nose Cone Cavity}

For the PLS storage ring with the beam stay-clear aperture of $100 \mathrm{~mm}$, the value of $R / Q$ is less than $50 \Omega$. Since the shunt impedance is directly proportional to the value 
Table 2: PLS LFS kicker dimensions

\begin{tabular}{lr}
\hline Parameter & Dimension [mm or ${ }^{\circ}$ ] \\
\hline \hline Inner cavity radius $R_{1}$ & 67.5 \\
Outer cavity radius $R_{2}$ & 101.5 \\
Port base radius $R_{3}$ & 95.1 \\
Cavity gap $d$ & 74.0 \\
Gap between cones $d_{1}$ & 64.0 \\
Barrier angle $\alpha$ & 24.0 \\
Port base angle $\beta$ & 16.9 \\
Back cavity size $b$ & 35.8 \\
Port base gap $g$ & 6.4 \\
Ridge length $\delta$ & 50.2 \\
Ridge distance $p$ & 87.2 \\
Back cavity distance $e$ & 123.0 \\
Port round radius $m$ & 2.0 \\
Port base round $h$ & 1.0 \\
\hline
\end{tabular}

Table 3: HOMs of the kicker

\begin{tabular}{lccc}
\hline Mode & $f[\mathrm{MHz}]$ & $R / Q[\Omega]$ & $Q$ factor \\
\hline \hline $\mathrm{TM}_{010}$ & 1130.5 & 49.0 & 21557.2 \\
$\mathrm{TM}_{011}$ & 2322.4 & 17.5 & 26524.4 \\
$\mathrm{TE}_{211}$ & 2503.0 & 17.3 & 40126.6 \\
$\mathrm{TM}_{111}\left(\mathrm{TE}_{011}\right)$ & 2749.4 & 5.6 & 44380.9 \\
$\mathrm{TM}_{211}$ & 3130.0 & 20.5 & 44898.5 \\
\hline
\end{tabular}

of $R / Q$, it is important to increase the value of $R / Q$ for higher shunt impedance, which depends on the geometry of pill-box. Therefore, it is possible to increase this value by change the geometry of the pill-box with help of SUPERFISH simulation. From the facts that the radius of beam chamber is fixed as $50 \mathrm{~mm}$ and that the central frequency of the kicker $f_{c}$ depends on the radius of outer cavity $R_{2}$, only the gap size $d$ can be adjustable to increase $R / Q$. Since the transit time factor $T$ and $R / Q$ are increased as the gap size $d$ is decreased, the shunt impedance (the transit time factor considered) will be increased by attaching a nose cone between a gap of pill-box cavity. The final pill-box cavity with nose cone attached has $255 \mathrm{MHz}$ of bandwidth and $1130.5 \mathrm{MHz}$ of central frequency which is near $1125 \mathrm{MHz}$. The final dimensions of the PLS LFS kicker are summarized in Table 2.

\subsection{Global performance of the kicker}

Investigation into the dangerous HOMs of the nose cone attached cavity is performed with the SUPERFISH and the result is summarized in Table 3. Frequencies of all dangerous HOMs are higher than the cutoff frequency of beam pipe ( $\sim 2295 \mathrm{MHz})$. By considering the fact that the singleridge of the waveguide widens the frequency separations between the HOMs, their frequencies are increased further beyond $2295 \mathrm{MHz}$. Therefore, they do not generate any undesirable CBMIs within the cutoff frequency of vacuum chamber. Thus, the kicker is free of dangerous HOMs. With 3D HFSS, the maximum value of gap voltage is 35.25

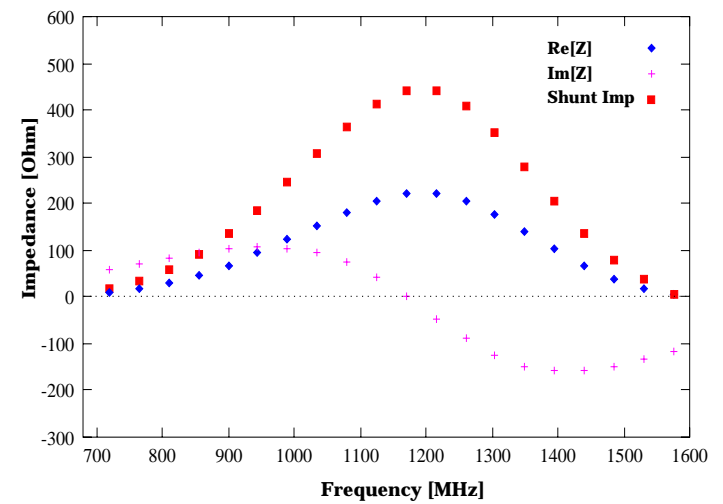

Figure 2: Measured $R_{s}$ and its frequency response

$\mathrm{V}$ for $1 \mathrm{~W}$ input power and its maximum shunt impedance is about $620 \Omega$ (transit time factor considered). The central frequency of the shunt impedance is about $1131 \mathrm{MHz}$ that is only $6 \mathrm{MHz}$ higher than the desired value of $1125 \mathrm{MHz}$.

\section{CURRENT STATUS}

With the LFS kicker design as described above, an aluminum kicker was fabricated by local manufacturer. After cleaning and assembly work was done, a series of performance test were followed with WILTRON 360B network analyzer. Measured central frequency and the bandwidth are $1115.6 \mathrm{MHz}$, and $344.4 \mathrm{MHz}$, respectively. The frequency response of the shunt impedance is shown in Fig. 2 with its maximum value of about $470 \Omega$. The $R / Q$ of measured HOMs within the cutoff frequency is lower than 3.3. So, they will not generate any dangerous CBMIs to the beam. This kicker will be installed to the PLS storage ring in April 1999 [3].

\section{ACKNOWLEDGMENTS}

The authors thank Dr. M. Tobiyama of KEK, Dr. D. H. Han of Kyungpook Univ., J. S. Yang, M. K. Park, Dr. J. Y. Huang, Dr. T. Y. Lee, Dr. M. H. Cho, and Dr. W. Namkung of PAL for their endless help and cheer.

\section{REFERENCES}

[1] M. Kwon et al, "The PLS RF System Upgrade Activities," Proc. of the First Asian Particle Accelerator Conference, (Tsukuba, Japan, March 1998), 749 (1999).

[2] R. Boni et al, "A Waveguide Overloaded Cavity as Longitudinal Kicker for the DA $\Phi$ NE Bunch-by-Bunch Feedback System," Particle Accelerator, 52, 95 (1996).

[3] Yujong Kim, J. Y. Huang, M. Kwon, and I. S. Ko, "Status of Longitudinal Feedback System for the PLS Storage Ring," In these proceedings. 